\title{
An Intelligent Railway Safety Risk Assessment Support System for Railway Operation and Maintenance Analysis
}

\author{
Min $\mathrm{An}^{*}$, Wanchang Lin, and Sheng Huang
}

School of Civil Engineering, The University of Birmingham, Birmingham B15 2TT, UK

\begin{abstract}
The paper presents the development of an intelligent railway safety risk assessment based support system. The proposed method can evaluate qualitative and quantitative safety risk data and information in a uniform manner for railway safety risk assessment. It permits the safety risk analysts to assess the risks associated with the failure modes directly using linguistic terms, i.e. qualitative descriptors. The proposed intelligent railway safety risk assessment system is capable of assessing the risks at component level, sub-system level and system level. It can assess not only "hard" risks (e.g. risks of a system), but also "soft" risks (e.g. staff risks). The outcomes of safety risk assessment are represented in two formats, risk score and risk category with a belief of percentage, which provide very useful safety risk information to railway designers, operators, engineers and maintainers for risk response decision making. An illustrative example of staff risk assessment in a railway depot is used to demonstrate the proposed intelligent railway safety risk assessment system. The results indicate that by using the proposed system, risks associated with a railway depot can be assessed effectively and efficiently.
\end{abstract}

Keywords: Railway safety, safety risk assessment, fuzzy reasoning approach, qualitative descriptors, staff safety risk assessment.

\section{INTRODUCTION}

Railways are by far one of the safest means of ground transportation, especially for their passengers and employees. However, there are serious issues involved in both maintaining this position in reality and sustaining the public perception of railway safety excellence [1-3]. The UK railway now finds itself in a situation where actual and perceived safeties are real issues, to be dealt with in a new public culture of rapid change, short-term pressures, and instant communications $[4,5]$.

The risk, in the railway industry, can be defined in relation to accidents and incidents leading to fatalities or injuries of passengers and employees. Recent structured hazard identification work within the industry has confirmed the high-risk scenarios of the types of accidents, such as collision, derailment and fire [4, 6, 7]. This shows the dangerous nature of the railway industry and demonstrates the need for increased awareness and better safety management. There are many possible causes of risk through operation and maintenance of vehicles and rail infrastructure and also from outside the railway such as vandalism and road incidents. Specifically, in the modification and maintenance of plain line, the largest number of serious incidences are from derailments and vehicles fouling infrastructure such as station platforms. There are many combinations of potential causes, each involving several disciplines and work-groups.

*Address correspondence to this author at the School of Civil Engineering, The University of Birmingham, Edgbaston, Birmingham B15 2TT, UK; Tel: +44-121-414-5146; Fax: +44-121-414-3698; E-mail: m.an@bham.ac.uk
Railway safety is a very complicated subject, which is determined by numerous aspects including human error. Many of the railway safety assessment techniques currently used are comparatively mature tools $[2,4,6,8-10]$. However, in many circumstances, the application of these tools may not give satisfactory results because the safety risk data are incomplete or there is a high level of uncertainty involved in the safety risk data. Therefore, it is essential to develop new safety risk analysis methods to identify major hazards and to assess the associated risks in an acceptable way under various environments where such mature tools cannot be effectively or efficiently applied. The safety information produced should be in a form to aid decisionmaking purpose. If risks are high, risk reduction measures must be applied and the operation and maintenance standards should be reassessed to reduce the occurrence probabilities and/or to control the possible consequences. If risks are negligible, no actions are required but the information produced needs to be recorded for audit purpose.

The quantified risk assessment (QRA) approaches such as fault tree analysis (FTA), event tree analysis (ETA) and equivalent annual fatality analysis (EAF) are currently used in railway safety risk analysis $[4,6,10]$, but often do not effectively handle with uncertainty of information as they rely heavily on the supporting statistical information that may not be available. Collecting sufficient data on which to base a statistical probability of the failure is a costly and difficult undertaking, and the relevance of data to any particular system, as well as its validity, is often questionable $[1,11]$. Furthermore, in many situations, the data of probability of failure of a system do not exist and it must be estimated based on the expert knowledge and experience or engineering judgement from similar items. In this context, a 
safety risk model using fuzzy reasoning-based approach may be more appropriate to analyse the risks of the system where there is incomplete safety risk information [3, 12, 13]. A fuzzy reasoning approach allows imprecision or approximate information in its analysis process, which helps to restore integrity to safety risk analysis. The fuzzy reasoning approach can handle imprecision, ambiguous, qualitative information and quantitative data in a uniform manner. It allows the safety risk analyst to evaluate the risk associated with the failure modes directly using qualitative descriptors that are more expressive and natural to describe the risk issues in railway safety risk assessment [14-16].

The paper presents an intelligent railway safety risk assessment system using a fuzzy reasoning approach. This system provides a structured way of combining the qualitative information with quantitative information from all available sources to facilitate safety risk analysis. The rest of the paper is organised as follows. In section 2, the fundamentals of fuzzy reasoning approach are described. The basic concepts of fuzzy set, membership function (MF), fuzzy operation, fuzzy IF-THEN rule and fuzzy inference system are outlined. Section 3 presents a methodology for the railway safety risk assessment using a fuzzy reasoning approach. The risk factors, such as failure probability (FP), consequent severity (CS), and risk level (RL) are discussed in terms of qualitative descriptors and how these qualitative descriptors are characterised within the MFs. The relationship between the risk factors and the RLs is addressed by the fuzzy IF-THEN rules base, which is based on engineering judgement and expert knowledge. Section 0 describes the implementation of the proposed intelligent railway safety risk assessment system. The structure of software is illustrated in block diagram and the function of each module is also presented in this section. In addition, the methodology of processing uncertainty in risk analysis is also discussed, which two pseudo codes are given to help the implementation of such processes. The proposed system not only assesses the RL of a single failure event of a railway component, but can also assess the risks at subsystem and system levels based on a set of failure events. An illustrative example of the staff safety risk assessment of a railway depot is presented in section 5 to demonstrate the effectiveness of the proposed intelligent system in railway safety risk analysis. The results of the safety risk assessment in the case example are represented as risk scores, located in a defined range, and risk category with a belief of percentage. Finally, section 6 gives conclusions and a summary of main benefits of using fuzzy reasoning approach in the railway safety risk assessment process.

\section{FUZZY REASONING APPROACH}

Fuzzy reasoning approach possesses the ability to mimic the human mind to effectively employ modes of reasoning that are approximate rather than exact. It enables the safety risk analyst to specify mapping rules in terms of qualitative expression rather than numbers and approximate function rather than exact reasoning.

\subsection{Fuzzy Sets and Membership Functions}

Fuzzy set was originally introduced by Zadeh [17]. A fuzzy set $A$ on a universe of discourse $U$ is defined as a set of ordered pairs [14, 17-19].

$$
A=\left\{\left(x, \mu_{A}(x)\right) \mid x \in U\right\}
$$

where $\mu_{A}(x)$ is called the MF of $x$ in $A$ that takes values in the interval $[0,1]$. The element $x$ is characterised by qualitative descriptors, for example, in railway safety risk assessment, one may often use such qualitative descriptors to present the FP as Improbable, Remote, Unlikely, Possible, Likely and Frequent; one may often use such qualitative descriptors to describe the CS as Minor, Major, Severe and Fatal; and qualitative descriptors such as Low, Possible, Substantial and High are used to describe the RL. Various types of MFs can be used, including triangular, trapezoidal, generalized bell shaped, and Gaussian functions [8]. However, the triangular and trapezoidal MFs are the most frequently used in safety risk analysis practice [1-3, 11-13].

Let $U$ be the universe of discourse $U=[0, u]$. A triangular MF can be defined as $A=\{a, b, c\}$

$f(x ; a, b, c)= \begin{cases}0, & x \leq a \\ \frac{x-a}{b-a}, & a \leq x \leq b \\ \frac{c-x}{c-b}, & b \leq x \leq c \\ 0, & c \leq x\end{cases}$

where three parameters $\{a, b, c\}$ with satisfaction of the relationship $a \leq b \leq c$ determine the $\mathrm{x}$ coordinates of three corners of a triangular MF.

Let $U$ be the universe of discourse $U=[0, u]$. A trapezoidal MF can be defined as $A=\{a, b, c, d\}$

$f(x ; a, b, c, d)= \begin{cases}0, & x \leq a \\ \frac{x-a}{b-a}, & a \leq x \leq b \\ 1 & b \leq x \leq c \\ \frac{d-x}{d-c}, & c \leq x \leq d \\ 0, & d \leq x\end{cases}$

Similarly, where four parameters $\{a, b, c, d\}$ with satisfaction of the relationship $a \leq b \leq c \leq d$ determine the $x$ coordinates of the four corners of a trapezoidal MF. The triangular and trapezoidal MFs are shown in Fig. (1).

A MF indicates the degree of preference. It should be noted that a numerical value, a range of numerical values and a triangular MF can be converted as simplified trapezoidal MFs, for example, when $a=b=c=d$, a MF is a numerical value; when $a=b$ and $c=d$, a MF is a range of numerical values; when $b=c$, a trapezoidal MF becomes a triangular $\mathrm{MF}$.

\subsection{Fuzzy Operations}

The union ("or") and intersection ("and") are two fuzzy operations, which are widely used in the safety risk assessment. The union of $A$ and $B$, denoted by $A \cup B$ or $A$ OR $B$, contains all elements in either $A$ or $B$, which is 

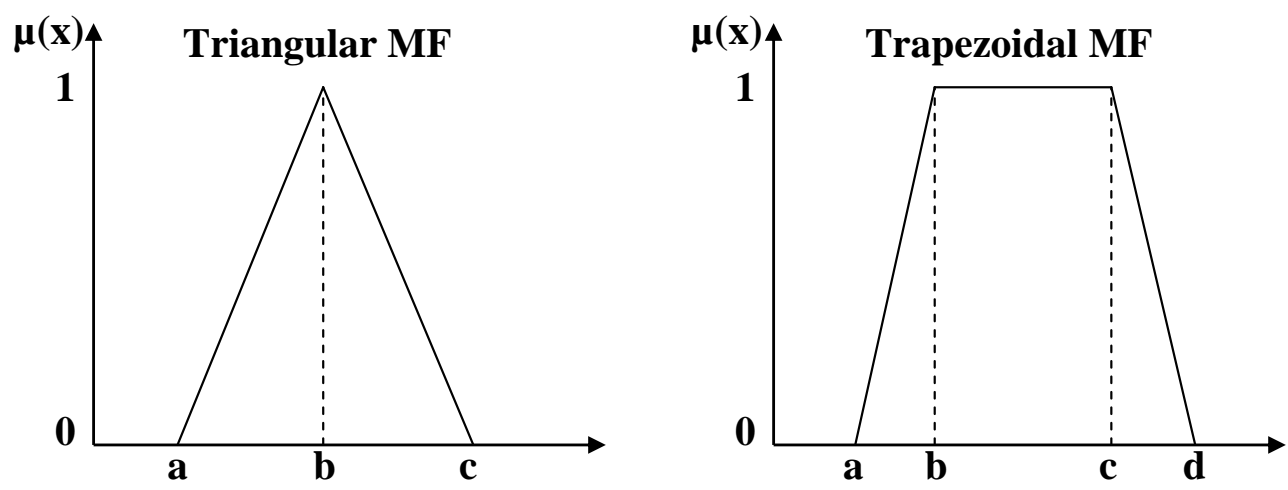

Fig. (1). Illustration of triangular and trapezoidal MFs.

calculated by the maximum operation and its MF is defined as:

$\mu_{A \cup B}(x)=\max \left\{\mu_{A}(x), \mu_{B}(x)\right\}$

The intersection of $A$ and $B$, denoted by $A \cap B$ or $A$ AND $B$, contains all the elements that are simultaneously in $A$ and $B$, which is obtained by the minimum operation and its MF is defined as

$\mu_{A \cap B}(x)=\min \left\{\mu_{A}(x), \mu_{B}(x)\right\}$

\subsection{Fuzzy IF-THEN Rule}

Fuzzy reasoning approaches are rule-based methodologies constructed from human knowledge in the form of fuzzy IF-THEN rules [3, 14, 18, 19]. A fuzzy IFTHEN rule is a statement in which some words are characterised by continuous MF. For example, the following is a frequently used fuzzy IF-THEN rule in railway safety risk assessment.

IF FP is likely and CS is fatal, THEN RL of the failure event is high.

where likely, fatal and high are qualitative descriptors characterised by MFs.

A fuzzy rule base consists of a set of fuzzy IF-THEN rules. Consider the input space $U=U_{1} \times U_{2} \times \cdots \times U_{n} \subset R^{n}$ and the output space $V \subset R$. Only the multi-input-singleoutput case is considered here, as a multi-output system can always be decomposed into a collection of single-output systems. Specifically, the fuzzy rule base comprises the follow fuzzy IF-THEN rules

$R_{i}$ : IF $x_{1}$ is $A_{1}^{i}$ and $\ldots$ and $x_{j}$ is $A_{j}^{i}$ and $\ldots$ and $x_{n}$ is $A_{n}^{i}$, THEN $y$ is $B^{i}$

$i=1,2, \ldots, r ; j=1,2, \ldots, n$

where $A_{n}^{i}$ and $B^{i}$ are the fuzzy sets in $U \subset R$ and $V \subset R$, respectively, and $x=\left(x_{1}, x_{2}, \ldots, x_{x}\right)^{T} \in U$ and $y \in V$ are the input and output qualitative descriptors of the fuzzy reasoning system, respectively. Owing to their concise form, fuzzy IF-THEN rules are often employed to capture the imprecise modes of reasoning that play an essential role in the human ability to make decisions in an environment of uncertainty and imprecision. Therefore, in the proposed fuzzy reasoning system, human knowledge has to be represented in the form of the fuzzy IF-THEN rules (Eq. (6)). There are three major properties of fuzzy rules that are outlined as follows $[2,20]$.

1. A set of fuzzy IF-THEN rules is complete if for any $x \subset U$, there exists at least one rule in the fuzzy rule base, say rule $R_{i}$ in the form of Eq. (4), thus

$$
\mu_{A_{j}^{i}}\left(x_{j}\right) \neq 0
$$

for all $j=1,2, \ldots, n$. Intuitively, the completeness of a set of rules means that at any point in the input space, there is at least one rule that 'fires', i.e. the membership value of the IF part of the rule at this point is non-zero.

2. A set of fuzzy IF-THEN rules is consistent if there are no rules with the same IF parts, but different THEN parts.

3. A set of fuzzy IF-THEN rules is continuous if there do not exist such neighbouring rules whose THEN part fuzzy sets have empty intersection, i.e. they do not intersect.

\subsection{Fuzzy Inference System}

The railway safety risk assessment system consists of two subsystems: fuzzy inference system (FIS) and user interface system. Fuzzy inference is to map from a given input to an output using fuzzy logic. The mapping provides a basis from which decisions can be made on the basis of both of qualitative and quantitative information. The process of fuzzy inference involves the developments of qualitative descriptors, MFs, fuzzy logic operations and fuzzy rule base [21-23]. The FIS as shown in Fig. (2) consists of four components: the fuzzy rule bases, fuzzification, fuzzy inference engine and defuzzification.

\subsubsection{Fuzzy Rule Base}

The development of the rule base involves various knowledge acquisition techniques to produce a body of information that could be useful in developing fuzzy qualitative descriptors and their associated MFs to qualify RLs. For many practical situations, several approaches can be used to gather information and knowledge required in deriving fuzzy rules. The knowledge acquisition methodologies used in this study include (a) historical data 


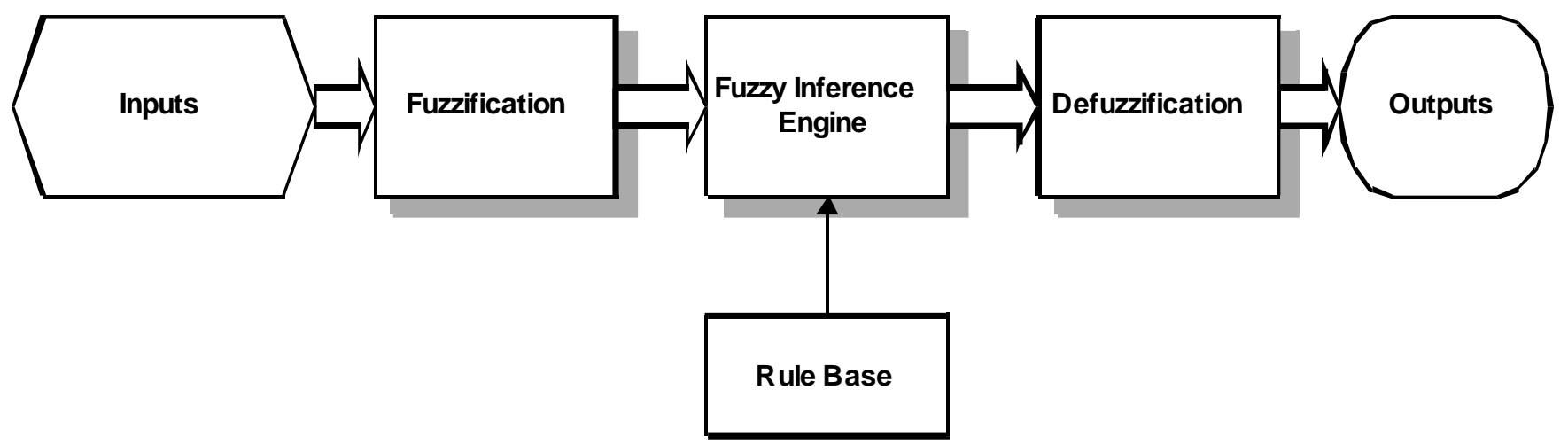

Fig. (2). Fuzzy inference system (FIS).

analysis, (b) failure analysis, (c) concept mapping, and (d) domain human expert experience and engineering knowledge analysis. These techniques are not mutually exclusive, and a combination of them is often the most effective way to determine the rule base $[3,11,13]$.

\subsubsection{Fuzzification}

The fuzzification converts input values into the corresponding fuzzy MF values. It determines the degrees of input values belonging to each of the appropriate fuzzy sets by MFs.

\subsubsection{Fuzzy Inference Engine}

- Evaluation of Fuzzy Rule. Once inputs have been fuzzified, these fuzzified values are employed to each rule to find out whether the rule will be fired. If a rule has true value in its premise, it will be fired and then contributes to the conclusion part. If the premise of a given rule has more than one part, the fuzzy operator is applied to evaluate the composite firing strength of the rule. Considering the $i$-th rule has two parts in the premise

$R_{i}$ : IF $x_{1}$ is $A_{1}^{i}$ and $x_{2}$ is $A_{2}^{i}$, THEN $y$ is $B^{i}$

$i=1,2, \ldots, r$

The two parts in the premise are connected with "and" and the firing strength $\alpha_{i}$ can be obtained using fuzzy intersection (minimum) operation

$\alpha_{i}=\min \left\{\mu_{A_{1}^{i}}\left(x_{1}\right), \mu_{A_{2}^{i}}\left(x_{2}\right)\right\}$

where $\mu_{A_{1}^{i}}\left(x_{1}\right)$ and $\mu_{A_{2}^{i}}\left(x_{2}\right)$ are the MFs of fuzzy sets $A_{1}^{i}$ and $A_{2}^{i}$.

- Implication. Implication is to shape the conclusion of a rule by using the firing strength obtained from the premise. In other words, the firing strength is implicated with the value of the conclusion MF by using fuzzy intersection operation and the output is a truncated fuzzy set. The implication using fuzzy intersection (minimum) operation is given by

$\mu_{i m p^{i}}(y)=\min \left\{\alpha_{i}, \mu_{B^{i}}(y)\right\}$ where $\mu_{B^{i}}(y)$ is the MF of conclusion part of a fuzzy rule and $\mu_{i m p^{i}}(y)$ is the MF of the truncated fuzzy set after implication.

- Aggregation. Aggregation is the process in which the truncated fuzzy sets that represent the implication outputs of each rule are aggregated into a single fuzzy set. The aggregation using fuzzy union (maximum) operation can by obtain by

$\mu_{\text {agg }}(y)=\max \left\{\mu_{i m p^{1}}(y), \mu_{i m p^{2}}(y), \cdots, \mu_{i m p^{r}}(y)\right\}$

where $\mu_{\text {agg }}(y)$ is the MF of the fuzzy set after aggregation.

\subsubsection{Defuzzification}

On the basis of the aggregated fuzzy set, defuzzification calculates the defuzzified value, which is a crisp value, standing for the final result of the fuzzy inference. The centroid of area method, which determines the centre of gravity of an aggregated fuzzy set, is the most frequently used method in the fuzzy reasoning systems $[3,8,11,13$, 23], defined as

$y_{d e f}=\frac{\int_{y} \mu_{a g g}(y) y d y}{\int_{y} \mu_{a g g}(y) d y}$

where $\mu_{a g g}(y)$ is the aggregated output MF.

\section{A RAILWAY SAFETY RISK ASSESSMENT MODEL}

The railway safety risk assessment model as shown in Fig. (3) includes the development of the qualitative descriptors for representing risk inputs, i.e. FP and CS, risk output, i.e. RL, and fuzzy rule base that presents the relationship between risk inputs and outputs. The qualitative descriptors are haracterized within fuzzy MFs. The fuzzy rule base is determined by data and failure analysis, human expert judgement, and engineering knowledge analysis. The $\mathrm{RL}$ of a failure event in the railway safety risk analysis is determined by two risk factors, i.e. FP and CS of a failure or hazard event $[2,4,6,12,13,15,16,25,26]$. 


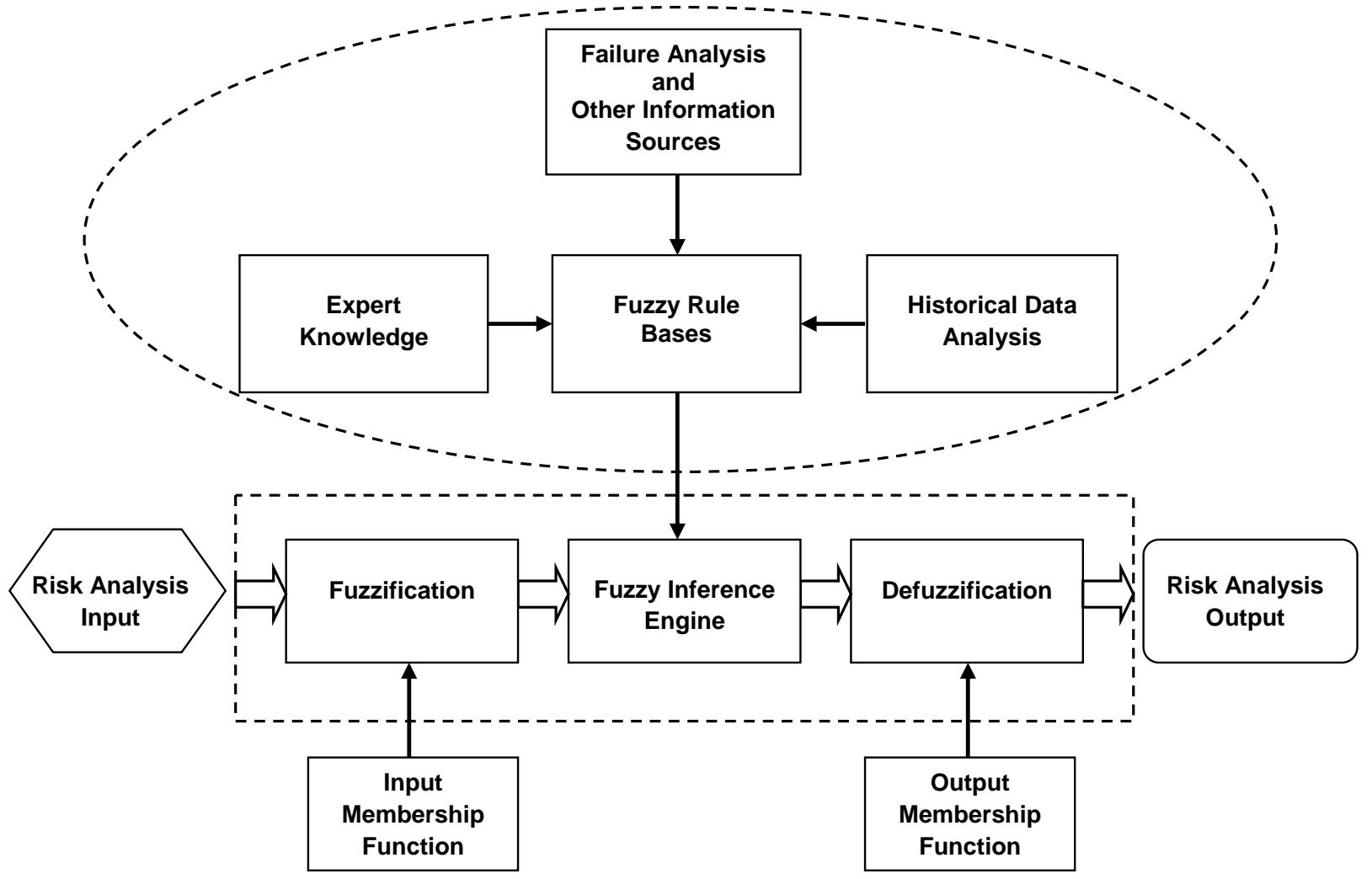

Fig. (3). Railway safety risk assessment model using fuzzy inference system.

\subsection{Qualitative Descriptors}

If a variable can take words in natural language as its value, it is called a qualitative descriptor, where the words are characterised by fuzzy sets defined in the universe of discourse in which the variable is defined. A qualitative descriptor is characterised by $(X, T, U, M)[2,3,15,16]$.

- $X$ is the name of the qualitative descriptor, for example, $X$ is FP of an item.

- $\quad T$ is the set of qualitative values that $X$ can take, for example

$T_{F P}=\{$ Improbable, Remote, Unlikely, Possible, Likely, Frequent\}

$T_{C S}=\{$ Minor, Major, Severe, Fatal $\}$

$T_{R L}=\{$ Low, Possible, Substantial, High $\}$

- $U$ is the actual physical domain in which the qualitative descriptor $X$ takes its quantitative (crisp) values, for example, $U=\left[F_{\text {Possible }}, F_{\text {Likely }}\right]$.

- $\quad M$ is a semantic rule that relates each qualitative value in $T$ with a fuzzy set in $U$, for example, $M$ relates Improbable, Remote, Unlikely, Possible, Likely and Frequent with the specific MFs of FP. Similarly, $M$ relates Minor, Major, Severe and Fatal with the specific MFs of CS, and Low, Possible, Substantial and High with the specific MFs of RL.

The concept of qualitative descriptors is important because qualitative descriptors are the most fundamental elements in human knowledge representation. When sensors are used to measure a variable, they give us numbers. When human experts are asked to evaluate a variable, they give us words. Hence, by introducing the concept of qualitative descriptors, it enables safety risk analysts to formulate vague descriptions in natural languages in precise mathematical terms. This will be able to incorporate human knowledge into engineering systems in a systematic and efficient manner.

Fuzzy qualitative descriptors are extensions of numerical variables in the sense that they are able to represent the condition of an attribute at a given interval by taking fuzzy sets as their values [1-3]. The values obtained in the development of fuzzy qualitative descriptors are considered as fuzzy-measuring attributes of objects, in this case, RLs.

The two fundamental parameters used to assess RL of a railway system on a subjective basis are FP and CS. Subjective assessment, for example, use of qualitative descriptors instead of ultimate numbers in probabilistic terms, is more appropriate to conduct safety risk analysis on these two parameters, as they are always associated with great uncertainty. Thus, these two parameters are represented by natural languages, which can be further described by the MFs. A MF is a curve that defines how each point in the input space is mapped to a membership value between 0 and 1. The fuzzy MFs are generated utilizing the linguistic categories identified in the knowledge acquisition and consisting of a set of overlapping curves.

The FP in terms of qualitative descriptors are defined as Improbable, Remote, Unlikely, Possible, Likely and Frequent. Based on the definitions used in the Workplace Risk Assessment (WRA) methodology [25, 26], according to 
the data collected from the railway industry, Table $\mathbf{1}$ describes the categories of FP, i.e. the number of times an event occurs over a specified period of time. For example, qualitative descriptor Remote is defined to cover the likelihood ranging from occurring once every ten years $(0.1)$ to occurring once every year (1.0) and qualitative descriptor Possible indicates likelihood ranging from occurring once every year (1.0) to occurring once every month (10). As the qualitative descriptors are categorized according to WRA values used in WRA $[25,26]$, the trapezoidal and triangular MFs are assigned to characterise these qualitative descriptors. The qualitative descriptors Improbable and Frequent are defined as trapezoidal MFs and others are defined as triangular MFs as shown in Fig. (4) and Table 1 shows the MF parameters.

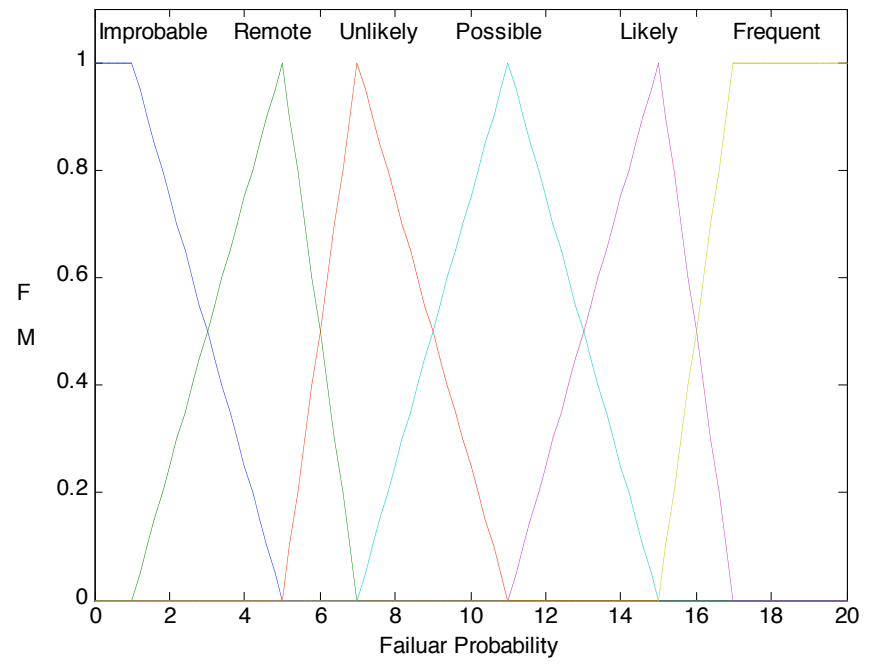

Fig. (4). MFs of failure probability.

The CS is described as Minor, Major, Severe and Fatal according to staff risk model used in WRA [25, 26]. The definitions of qualitative descriptors about CS are given in terms of the number of fatalities, major and minor injuries resulting from the occurrence of a particular hazardous event as shown in Table 2 . In this study, minor and major injuries are equated to fatalities, i.e. a minor injury is equated to 0.001 fatalities, a major injury to 0.01 fatalities and severe injury to 0.1 fatalities. The qualitative descriptors Minor and Fatal are defined as the trapezoidal MFs while Major and Sever as triangular MFs as shown in Fig. (5). Their MF parameters are chosen according to WRA values [16, 17] and listed in Table 2.

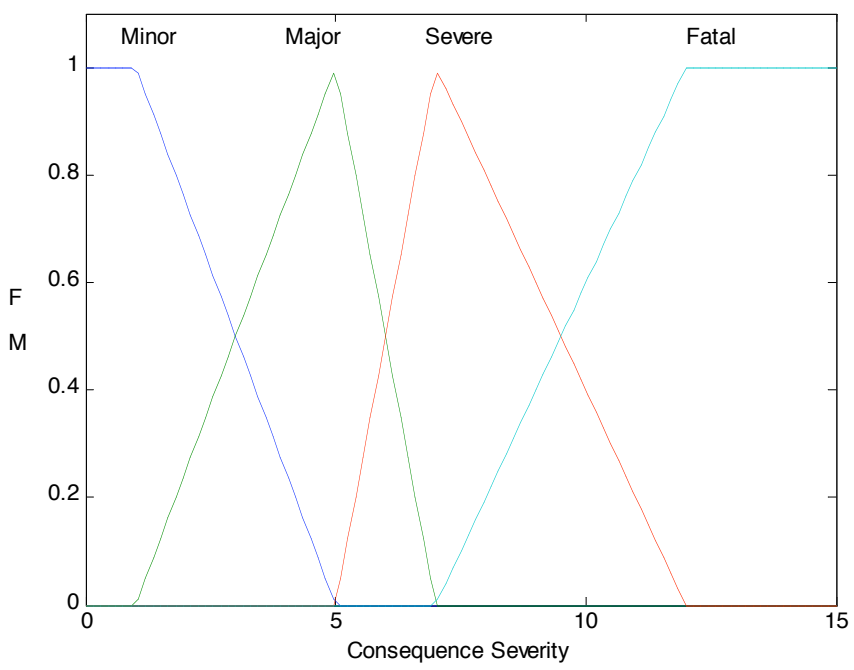

Fig. (5). MFs of consequent severity.

The fuzzy set of RL in terms of qualitative descriptors is defined as Low, Possible, Substantial and High [3, 12, 13, 15, 16]. Their definitions are generally similar to those described in EN50126, EN50129, and GE/GN8561 [5, 24, 27] are listed in Table $\mathbf{3}$. The risk score is defined in a manner that the lowest score is 0 , where as the highest score is 10. For example, qualitative descriptor Low is defined on the basis of the risk score ranging from 0 to 1 . Similar to the input qualitative descriptors of FP and CS, the trapezoidal MFs are used to describe the RL as shown in Fig. (6). The result of RLs can be expressed either as risk score located in the range from 0 to 10 or as risk category with a belief of percentage.

\subsection{Fuzzy Rules}

Fuzzy rule base consists of a set of fuzzy IF-THEN rules. It is the core of a fuzzy logic system in the sense that all other components are used to implement these rules in a reasonable and efficient manner. The fuzzy rules as described in Section 2 are basically built through the study of engineering knowledge, historical incident, and accident information. The human experts have a good intuitive knowledge of the system behaviour and risks involved in various types of failures. As the fuzzy rules are linguistic rather than numerical, they provide a natural framework for expressing human knowledge. Thus, experts often find fuzzy rules to be a convenient way to express their knowledge about the relationship between input and output variables. These sources are not mutually exclusive and a

Table 1. Qualitative Descriptors of Failure Probability

\begin{tabular}{|c|c|c|c|c|}
\hline Qualitative Descriptors & Description & Likelihood (Event/Year) & WRA Value & MF Parameters \\
\hline \hline Improbable & $<1$ in 100 years/ extremely unlikely & $<0.01$ & 1 & $0,0,1,5$ (Trapezoid) \\
\hline Remote & 1 in 10 years to 1 in 1 year & $0.1-0.3$ & 7 & $5,7,7$ (Triangle) \\
\hline Unlikely & 1 in 1 year to 1 in 10 years & $0.3-1$ & 11 (Triangle) \\
\hline Possible & 1 in 1 year to 1 in 1 month & $1-10$ & 15 & $11,11,15$ (Triangle) \\
\hline Likely & 1 in 1 month to 1 in 1 week & $10-50$ & 17 (Triangle) \\
\hline Frequent & $>1$ in 1 week & $>50$ & $15,17,20,20$ (Trapezoid) \\
\hline
\end{tabular}


Table 2. Qualitative Descriptors of Consequent Severity

\begin{tabular}{|c|c|c|c|c|}
\hline Qualitative Descriptors & Description & Equivalent Fatalities (EF) & WRA Value & MF Parameters \\
\hline \hline Minor & $<3$ days off work & 0.001 & 1 & $0,0,1,5$ (Trapezoid) \\
\hline Major & Between 3 days and 1 months off work & 0.01 & 5 & $1,5,7$ (Triangle) \\
\hline Severe & $>1$ month off work & 0.1 & 12 & $7,7,12$ (Triangle) \\
\hline Fatal & Fatality & 1 & $7,15,15$ (Trapezoid) \\
\hline
\end{tabular}

combination of them is often the most effective way to determine the rule base.

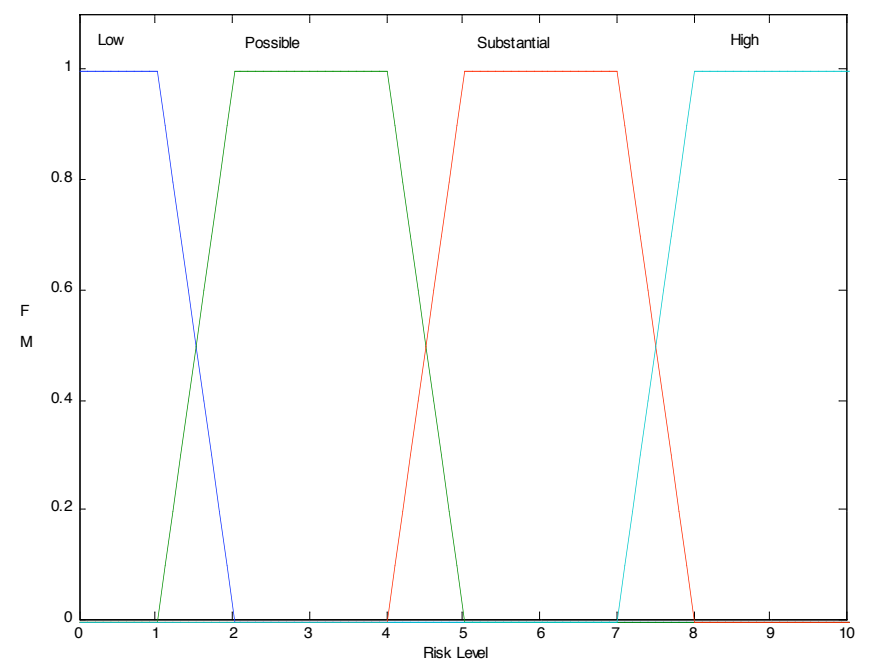

Fig. (6). MFs of risk level.

Several factors also have an influence in developing the fuzzy rule base as follows [2, 3, 12, 13].

- $\quad$ Completeness. The fuzzy rule base should cover all matches between inputs and outputs.

- Number of rules. Although there is no general procedure for deciding the optimal number of rules, the decision should consider importance of performance, efficiency of computations and choice of qualitative descriptors.

- Consistency and correctness. The choice of fuzzy rules should minimise the possibility of contradiction and unwanted interactions between the rules.

In this study, as FP has six qualitative descriptors and CS has four qualitative descriptors, their combination, connected with "and", leads to total twenty-four rules, as listed in Table 4. These rules are subjectively defined based on the expert experience and engineering judgment. For example, the rule at bottom left of Table 4 would be expressed as follows.

IF failure probability is improbable and consequent severity is minor THEN risk level is low.

There are two parts combined through an intersection operator "and" in the premise of the above rule. If the premise has a true value via an "and" operator, the premise will be fired and will contribute to the fuzzy conclusion, in this case, RL. If the premise is true to some degree of membership, then the conclusion is also true to that same degree.
The importance of fuzzy IF-THEN rules stems from the fact that human expertise and knowledge can often be represented in the form of fuzzy rules [2, 3, 12, 13]. Rules based on these types of qualitative descriptors are more natural and expressive than numerical numbers and calculations. The fuzzy rules also allow quantitative data such as the FP, and qualitative or judgemental data such as severity of consequence to be combined in a uniform manner for the RL.

\section{IMPLEMENTATION OF THE INTELLIGENT RAILWAY SAFETY RISK ASSESSMENT SYSTEM}

\subsection{System Module Structure}

An intelligent railway safety risk assessment system based on the proposed approach has been developed. The system has been written for a PC platform in $\mathrm{C}++$ and operates under Windows 98, 2000, 2007 and XP. The modular structure of the proposed intelligent system is shown in Fig. (7), which consists of two main modules, i.e. fuzzy inference system (FIS) and Graphical user interface (GUI):

\section{Fuzzy inference system}

This module implements the FIS as described in Section 0 and consists of the following four sub-modules:

(a) Fuzzification. The fuzzification converts the crisp inputs of FP and CS into fuzzified inputs located between 0 and 1 with respect to the corresponding $\mathrm{MF}$. The input can be a numerical value or an interval value that indicates the input uncertainty.

(b) Implication. The implication is to shape the conclusion part of fired rule from the premise part to the conclusion part of a fired or active rule by using the fuzzy "and" operator. Evaluation of fuzzy rules is to determine which rule in the rule base is fired or not. If a rule has a true value in its premise, it will be fired and then contributed to the conclusion part.

(c) Aggregation. Aggregation is the process to synthesize the fuzzy sets, which represents the outputs of all fired rules into a single fuzzy set by using fuzzy "or" operator.

(d) Defuzzification. Defuzzification is to convert the aggregated results produced from aggregation to a crisp output that represents the final result of fuzzy inference, i.e. risk score and risk categories with a belief of percentage.

\section{Graphical user interface}

This module implements the interface for the data exchange between the user and the computer, which includes the following sub-modules: 


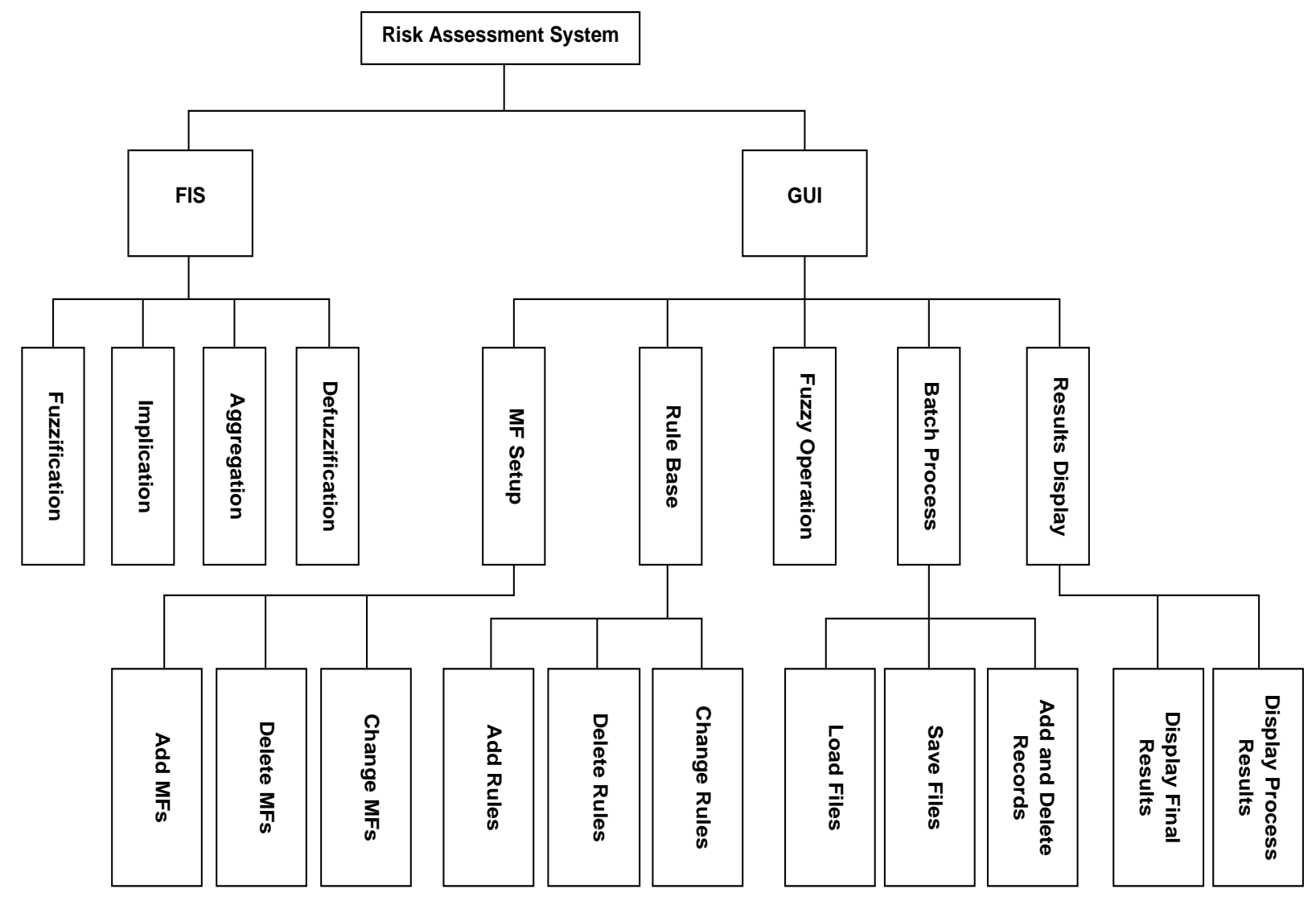

Fig. (7). Risk assessment system model structure.

(e) MF Setup. This module enables users to set up qualitative descriptors, MFs and input values. It consists of Add MF, Delete MF and Change MF functions for the MF construction and maintenance.

(f) Rule Base. This module contains fuzzy rules. The construction of a fuzzy rule is based on the qualitative descriptors developed in the MF setup module and connected with "and" or "or" operator. The functions of Add Rule, Delete Rule and Change Rule enable the user to add new fuzzy rules, delete the existed fuzzy rules and change the behaviours of fuzzy rules from the rule base.

(g) Fuzzy Operation. The module sets up fuzzy operations required in the FIS including fuzzy "and", "or", implication, aggregation and defuzzification.

(h) Batch Process. Users can assess the RL of a set of failure events and subsystems and/or a system. A set of input values can be added, changed or deleted via a grid control by using Add and Delete Record functions. The establishment of a set of events can be saved into a text file and the file can also be loaded again into the system by using Save File and Load File functions.

(i) Results Display. This module can display the results of implication, aggregation and final RL. For example, Display Final Results function shows the risk score and risk categories with a belief of percentage, and Display Process Results function shows the results of implication and aggregation.
The main benefit of using this type of structure is that the data are completely separated from the users. This independence enables modifications to be made to each of the modules individually with little or no impact on the others. This is useful if, for example, certain MFs within the system need updating, as it can be done without affecting the behaviour of the other modules.

In railway safety risk assessment, the input values of FP and CS are required. As stated earlier in this article, because the process of risk analysis is very complex and data available may be incomplete for risk assessment in many circumstances, it may be extremely difficult to conduct traditional probabilistic risk analysis to assess the occurrence likelihood of hazards and the magnitude of their possible consequences because of the great uncertainty involved. For example, assume the input value of FP is 8 as indicated by the vertical line as shown in Fig. (8), the input is fuzzified to be Unlikely and Possible with a belief of $75 \%$ and $25 \%$, respectively. These MF values are then used in safety risk assessment process. However, even with detailed guidelines, it is often difficult to translate feelings and experience into a number that represents exactly how much more one parameter impacts on a given event than other. In this case, as described in the Fuzzification module, if the exact value of a FP or CS is not acquired, an interval value can be used as uncertain input. The uncertainty is processed in a manner that the values near the centre of interval are assumed to be more certain than those near the edges, and the width of the interval indicates the amount of uncertainty in the input. For example, the FP of an event is around 9 to 16 and most likely to be 13 in the universe of $(0,20)$. The input values of FP are 
interval as shown in Fig. (8). Therefore, the values near the centre of interval are assumed to be more certain than those near the edges. Such an interval input is associated with a triangular MF. The points of intersection between the MF of interval input and the relevant $\mathrm{MF}$ of input qualitative descriptors are treated as the fuzzified inputs. As shown in Fig. (8), in this case, the input of FP lies in the range from 9 to 16 and mostly to be 13 associated with a triangular MF. It can be seen that the interval input has intersection points with qualitative descriptors Unlikely, Possible, Likely and Frequent. It indicates that this interval input belongs to these four categories of FP with different MF values. The values of intersection points between the interval input and the fuzzy MF are the solutions of a non-linear equation below

$f(x)=\mu_{A}(x)-\mu_{B}(x)=0$

where $\mu_{A}(x)$ and $\mu_{B}(x)$ are the MF of the interval input and a qualitative descriptor, respectively. The numeric analysis approach, secant method, has been employed to calculate intersection points [14].

Assume two initial approximations $p_{0}$ and $p_{1}$, the following equation is used to calculate the iterative solutions of $f(x)=0$ :

$p_{k+1}=p_{k}-\frac{f\left(p_{k}\right)\left[p_{k}-p_{k-1}\right]}{f\left(p_{k}\right)-f\left(p_{k-1}\right)} \quad$ for $k=1,2, \cdots$

where $k$ is the index of iteration.

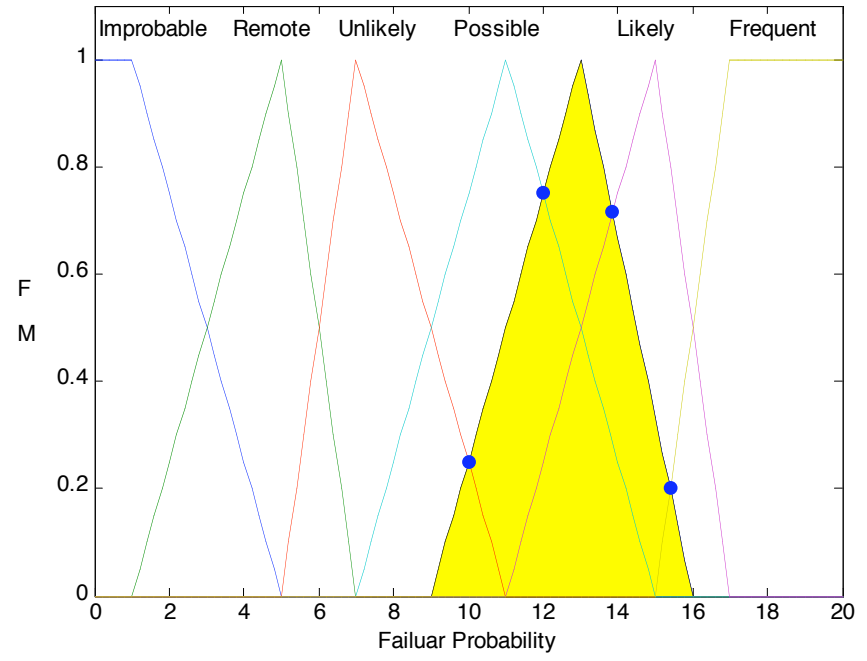

Fig. (8). MFs of failure probability.

The following pseudo codes describe the uncertainty processing:

PROCEDURE uncertainty processing

\section{BEGIN}

FOR $i:=1$ to No. of MFs

CALL: Secant-method (uncertainty input, current MF)

IF find intersection THEN

Store them

ENDIF

ENDFOR

END
For example, as illustrated in Fig. (8), the interval input has one intersection point withMFs of Unlikely, Possible, Likely and Frequent. As the two functions are stepwise linear functions, the value pairs, $(5,6),(6,7),(7,8),(8,9),(9,10)$, $(10,11),(11,12),(12,13),(13,14),(14,15),(15,16),(16$, $17),(17,18),(18,19)$ and $(19,20)$, are chosen as the initial values. Fifteen sessions of calculation are executed on the basis of the fifteen pairs of the initial values to find intersection points between the interval input and the qualitative descriptors Unlikely, Possible, Likely and Frequent. In this case, there are four intersection points: (10, $0.24),(11.8,0.76),(14.1,0.71)$ and $(15.5,0.18)$. In other words, the inputs are fuzzified to be Unlikely with a belief of $24 \%$, Possible with a belief of $76 \%$, Likely with a belief of $71 \%$ and Frequent with a belief of $18 \%$. Similarly, the uncertainty associated with CS can also be traded using equations (16) and (17). These fuzzified input values are then used by the FIS to assess the RL.

As described earlier in this section, the Batch Process module deals with risk assessment from component level, then progressing up to the subsystem level and finally to the system level. The pseudo codes for risk assessment at subsystem and/or system level are listed as follows

PROCEDURE system level risk assessment

\section{BEGIN}

\section{REPEAT}

Read a failure event.

Run main FIS operation.

Get risk level of component level.

Get the aggregation results of this failure event.

Combine these aggregation results with previous one.

UNTIL reach the final failure event.

Perform defuzzification on the combined aggregation results.

END

\subsection{Application of the Proposed Intelligent System for Railway Safety Risk Assessment}

Some screen shots of the proposed intelligent railway safety risk assessment system are shown in Figs. (9- 12). The proposed intelligent system can be used to assess the risk of a failure event with quantitative and qualitative information, the risks of a set of failure events and sub-systems and/or a system. Using GUI, qualitative descriptors and their MFs can be easily set up. Based on these qualitative descriptors, the rule base can be established correspondingly. Once the qualitative descriptors, MFs and rule base have been set up, the system is ready to process safety risk analysis. The system consists of a number of tab pages to deal with the MF parameter setup, rule base development, fuzzy operation selection, process result and final result display, which are described as follows.

- $\quad$ Failure Probability Tab. Users can set up qualitative descriptors of FP, types of MFs and their corresponding parameters as shown in Fig. (9). The command bottoms, Add MF, Delete $M F$ and Change 
$M F$, provide editing functions for MFs development. When the user enters the parameters of a MF, the system will check the validity of the parameters automatically. If they do not satisfy the required conditions, an error message box will prompt the user to check the parameters and enter the correct parameters. The text box of Value allows the user to enter the value of FP. For example, when a numerical value of 7 is input into the text box of Value and a vertical line as shown in Fig. (9) in the graphical window of Membership Function Plot indicates such an input. In some cases, where no exact value of FP is known, the user can input two values separated by a comma as an interval input to indicate the uncertainty. The interval values will be shown in the graphical window of Membership Function Plot with a triangular $\mathrm{MF}$.

- $\quad$ Consequent Severity Tab. This tab has the exact same functions as Failure Probability Tab to set up the qualitative descriptors and their MFs of CS.

- $\quad$ Risk Level Tab. Similar to Failure Probability Tab and Consequent Severity Tab, this tab enables the user to set up the qualitative descriptors and MFs of RL.

Rule Base Tab. This tab is used to construct fuzzy rules based on qualitative descriptors of FP, CS and RL expression. For example, when the user adds a new rule as shown in Fig. (10), the input qualitative descriptor of Failure Probability from Inputs combo box can be selected and then one of qualitative values will be added to the box of If. Similarly, the qualitative descriptor of Consequent Severity can be selected and its value will be added to the If box with a connection of OR/AND by clicking the radio button. The above two steps are used to construct the premise part of a fuzzy rule. Then an output qualitative value from the box of then output is, i.e. Low, Possible, Substantial and High can be selected. Finally, by pressing Add Rule button to add the rule into rule base which will be shown in the box of Rules. If a rule needs to be deleted from the rule base, the user can select such a rule from the box of Rules and then press Delete Rule button to do so. If a rule needs to be edited from the rule base, the user can select such a rule from the box of Rules, make necessary change and then press Change Rule button.

Project Tab. The user can choose fuzzy operations such as AND, OR, Implication, Aggregation and Defuzzification from the corresponding combo boxes as shown in Fig. (11). The text box of Name shows current project name. The user can change the project name on the basis of particular cases. When all of the parameters are set up, the results are then shown in the result windows by pressing command button Run. The graphical window of Final Result shows the MFs of RL with the defuzzified value and its percentages belonging to the defined qualitative descriptors. By pressing Previous Result and Next Result buttons, the user can see the firing strength and its implication results of each rule. The graphical window of
Progress Results illustrates the results of implication and aggregation.

- Data Grid Tab. Users can add the values of FP and CS of failure events to the data grid, change or delete them via this tab. These values of FP and CS, can be saved into a comma-separated-value (CSV) file by pressing command button Save. When the values of FP and CS are needed to be exported from other data format, such as Excel format, into a CSV file, the system is able to load such a data file into the data grid tab by pressing command button Load. Once the values of FP and CS have been loaded into the data grid tab, by pressing command button Run and the RL of each failure event is displayed in the column of Risk Level as shown in Fig. (12). Also the RLs of subsystem and/or a railway system with respect to all of these failure events are computed and listed in the column of Risk Level of System.

\section{AN ILLUSTRATIVE EXAMPLE: METRONET STAFF RISK ASSESSMENT}

An illustrative example of staff risk assessment is used to demonstrate the proposed intelligent railway safety risk assessment system using fuzzy reasoning approach. The input parameters are FP and CS of hazardous events. The outputs of safety risk assessment are the RLs of specific hazards, hazard groups and a railway depot. The hazard group RLs are produced using the fuzzy reasoning approach based on the aggregation results of each specific hazard belonging to the particular hazard group. The RL of the railway depot is computed based on the aggregation of the RLs of each hazard group. The format of output has two forms. One is risk score located from 0 to 10 and other one is risk categorized as Low, Possible, Substantial and High with a belief of percentage. The data set has been collected from the industry and the example has been involved to assess the risk to staff in a typical depot, Northumberland Park, in which a number of hazard groups are defined as $[19,26]$

- Electricity: The specific hazards include arcing eye, arcing from $630 \mathrm{~V}$, attaching or removing targets, contact with $630 \mathrm{~V}$ during task, contact with low voltage, gaining entry to unit, removing or inserting 630 volt jumpers, walking in pit, walking near live track, and so on.

- $\quad$ Falling Objects: The specific hazards include stacked items, tools and train parts.

- $\quad$ Fire/Explosion: The specific hazards include burning equipment, gas cylinder and hose leakage, ignition of flammable gases and ignition of flammable solvent.

- Hand Tools: The hazards include ejection of material from hand tools, entanglement with hand tools and minor injury from hand tools.

- Health Hazards: The specific hazards include glazing, corrosive substance, irritant substance, noise/ vibration, particulates/dust, toxic substance, and so on.

- Machinery: The specific hazards include compressed air, crushing, ejection of material from machinery, entanglement with machinery and minor injury from machinery. 


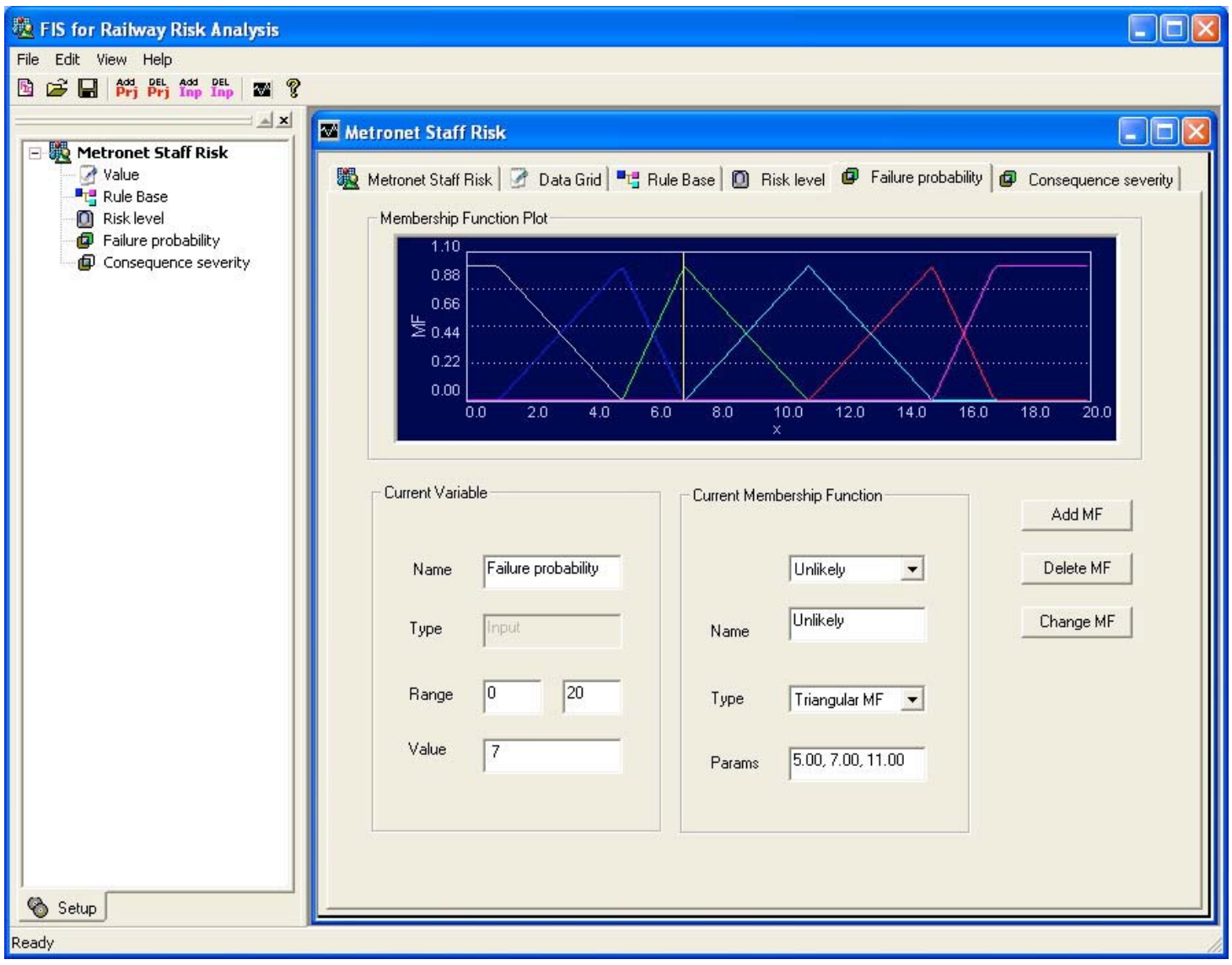

Fig. (9). Failure probability tab.

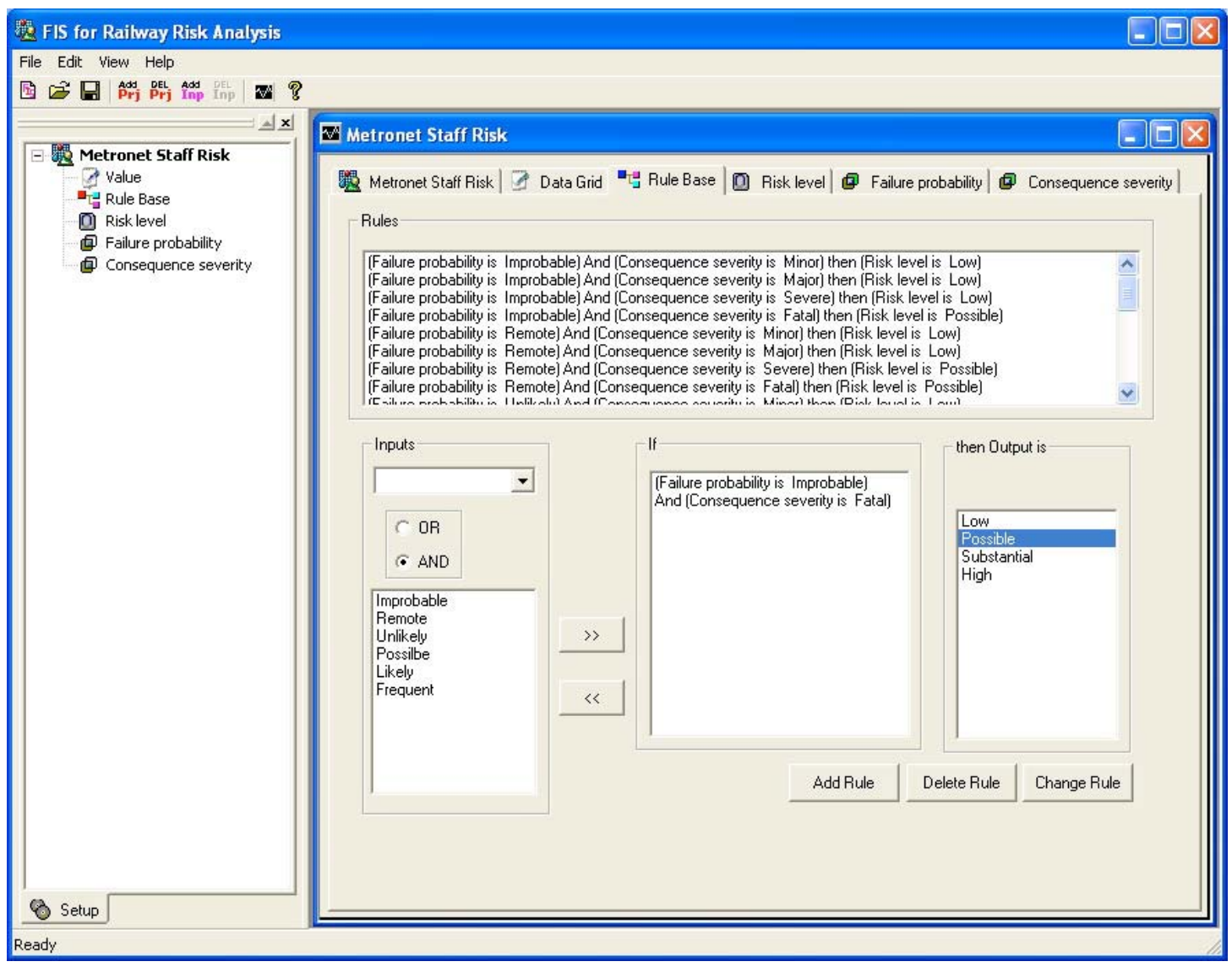

Fig. (10). Rule base tab. 


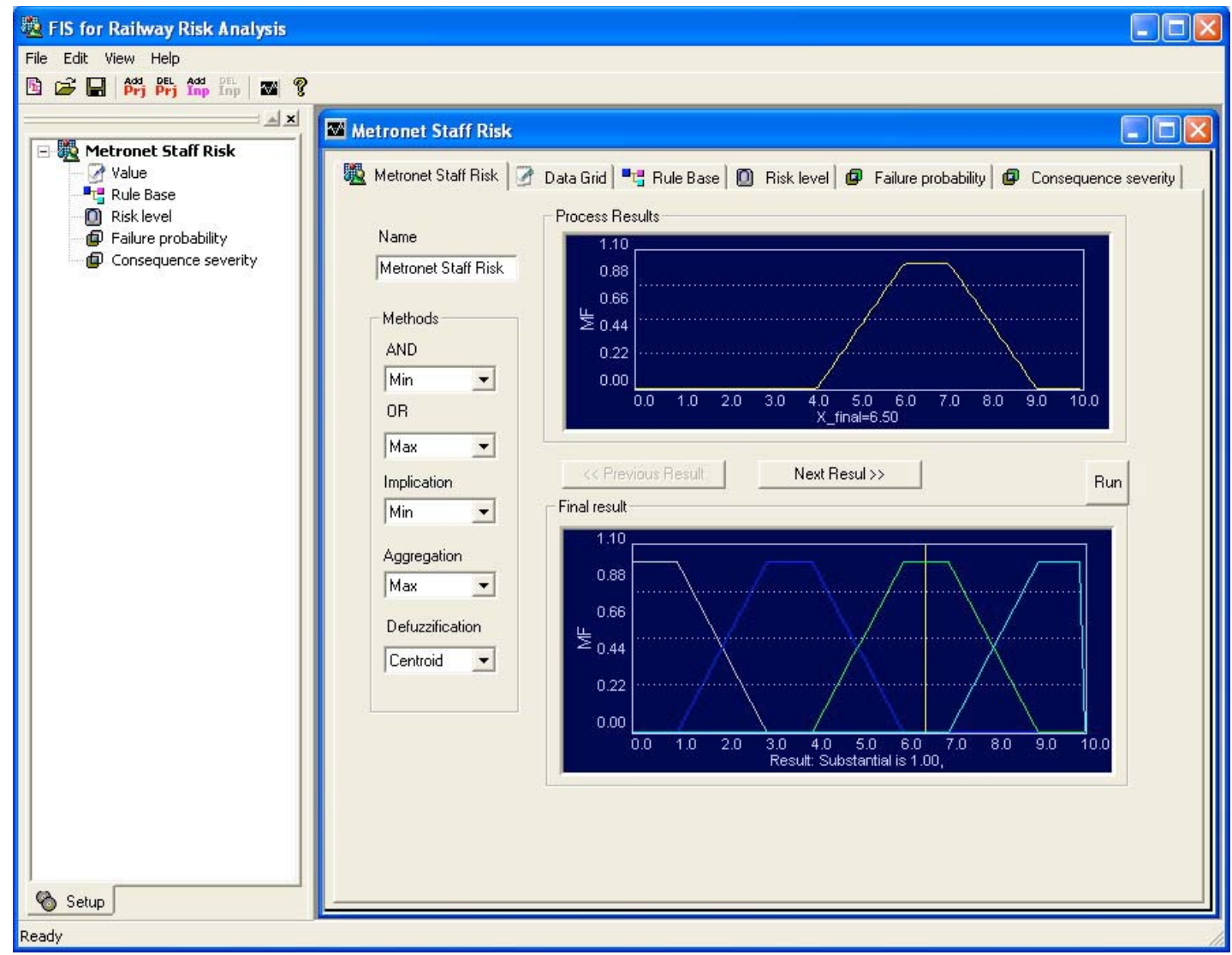

Fig. (11) Risk level tab.

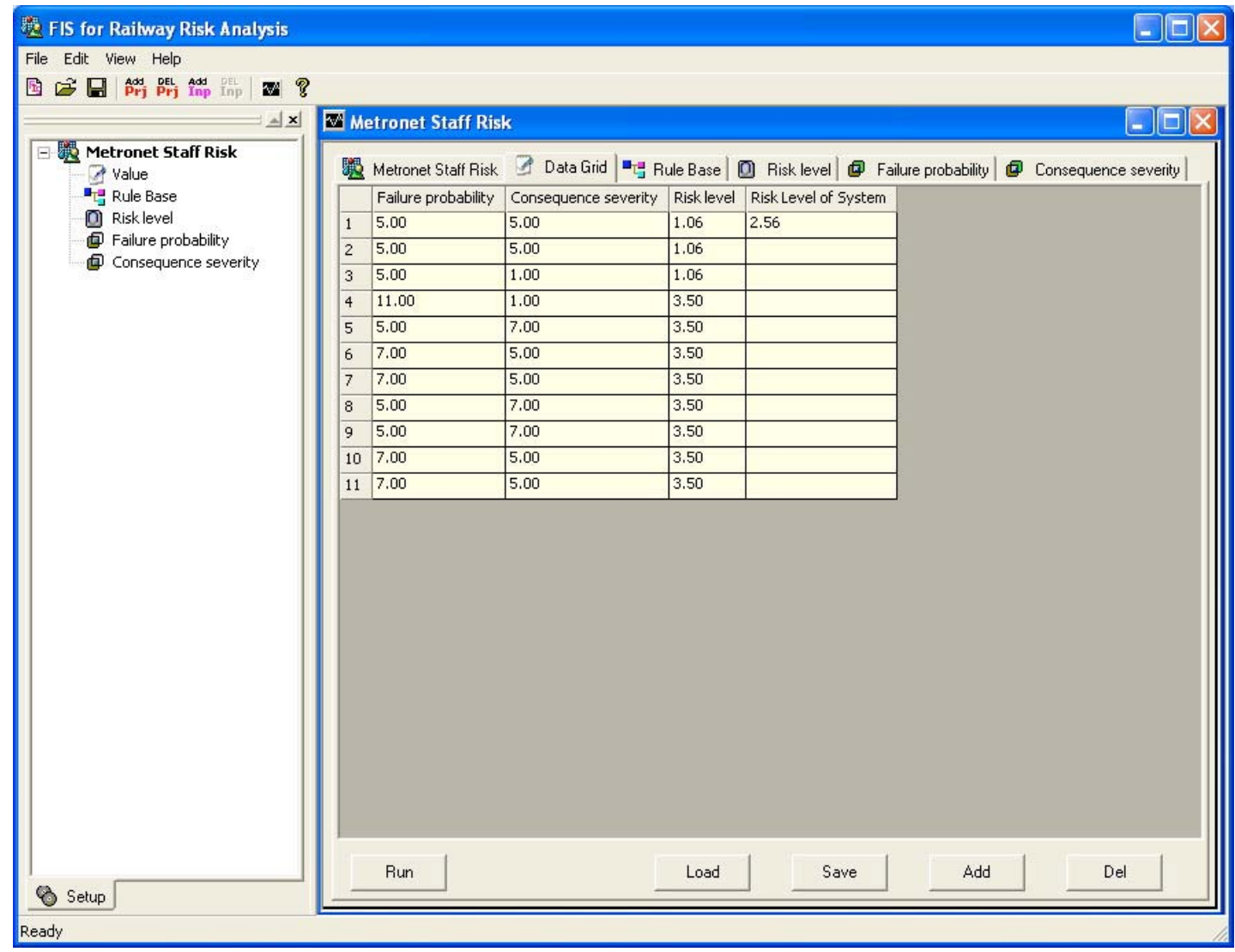

Fig. (12). Data grid tab. 
- $\quad$ Manual Handling: The specific hazards include changing train parts, lifting loads, maneuvering loads, operating machinery and contacting overhead $630 \mathrm{~V}$ jumper.

- Slips, Trips \& Falls: The specific hazards include access/egress from pit, access/egress from unit, attaching/removing targets (above the pit), attaching/ removing targets (in the pit), descending/climbing stairs, driving vehicles near the pit, from height (e.g. ladders), from unit during task, through opening trap door/manhole, walking around depot and walking around depot (into the pit).

- Vehicle Collision: The specific hazards include collision with another train, collision with plants/people and collision with uncontrolled movement of units.

Each hazard group is divided into specific hazards, which consists of a large number of failure events. These specific hazard events have been saved as Excel data files which can be read directly by the proposed intelligent railway safety risk assessment system.
The qualitative descriptors of FP and their MFs are defined as Improbable, Remote, Unlikely, Possible, Likely and Frequent as shown in Fig. (4). The CS is described as Minor, Major, Severe and Fatal which are characterised by triangular and trapezoidal MFs as shown in Fig. (5). The qualitative descriptors of FP and CS have been used to assess RL of each specific hazard. The results are listed in Table 6. The qualitative descriptors of RL and their MFs are defined as Low, Possible, Substantial and High as shown in Fig. (6). The RL of each specific hazard event obtained is represented by a risk score ranging from 0 to 10 and the risk categories defined in Table $\mathbf{3}$ with a belief of percentage belonging to these categories. Table 4 gives the fuzzy rules to describe the relationships between the FP, CS and RL, which has been used to assess RLs of staff risks at Northumberland Park Depot. The hazard group RLs are produced using the fuzzy reasoning approach based on the aggregation results of each specific hazard belonging to the particular hazard group. The $\mathrm{RL}$ of the railway depot is computed based on the aggregation of the RLs of each hazard group.

Table 3. Qualitative Descriptors of Risk Level

\begin{tabular}{|c|c|c|c|}
\hline Qualitative Descriptors & Description & Risk Scores & MF Parameters \\
\hline \hline Low & Review subject to availability. & $0-1$ & $0,0,1,3$ (Trapezoid) \\
\hline Possible & Review to be carried out and corrective action implemented. & $3-4$ & $1,3,4,6$ (Trapezoid) \\
\hline Substantial & Review and corrective action to be carried out. & $6-7$ & $4,6,7,9$ (Trapezoid) \\
\hline High & Need immediate corrective action. & $9-10$ & $7,9,10,10$ (Trapezoid) \\
\hline
\end{tabular}

Table 4. Rule Base of Staff Risk Assessment

\begin{tabular}{|c|c|c|c|c|c|c|}
\hline \multirow{2}{*}{ Consequent Severity (CS) } & \multicolumn{5}{|c|}{ Failure Probability (FP) } \\
\cline { 2 - 7 } & Improbable & Remote & Unlikely & Possible & Likely & Frequent \\
\hline \hline Fatal & Possible & Possible & Substantial & Substantial & High & High \\
\hline Severe & Low & Possible & Possible & Substantial & Substantial & High \\
\hline Major & Low & Low & Possible & Possible & Substantial & Substantial \\
\hline Minor & Low & Low & Low & Possible & Possible & Substantial \\
\hline
\end{tabular}

Table 5. Risk Calculation of Hazard Groups at Northumberland Park Depot

\begin{tabular}{|c|c|c|c|}
\hline Hazard Groups & WRA Records & Risk Scores & Risk Categories \\
\hline Electricity & 380 & 2.56 & Low: $22 \%$, Possible: $78 \%$ \\
\hline Falling Objects & 32 & 2.56 & Low: $22 \%$, Possible: $78 \%$ \\
\hline Fire / Explosion & 12 & 1.06 & Low: $97 \%$, Possible: $3 \%$ \\
\hline Hand Tools & 35 & 2.56 & Low: $22 \%$, Possible: $78 \%$ \\
\hline Health Hazards & 72 & 4.07 & Possible: $97 \%$, Substantial: $3 \%$ \\
\hline Machinery & 64 & 4.07 & Possible: $97 \%$, Substantial: $3 \%$ \\
\hline Manual Handling & 178 & 4.07 & Possible: $97 \%$, Substantial: $3 \%$ \\
\hline Slips, Trips \& Falls & 493 & 2.56 & Low: $22 \%$, Possible: $78 \%$ \\
\hline Vehicle Collision & 24 & 2.56 & Low: $22 \%$, Possible: $78 \%$ \\
\hline Railway Depot: & 1290 & 4.07 & Possible: $97 \%$, Substantial: $3 \%$ \\
\hline
\end{tabular}


Table 6. Risk Calculation of Specific Hazards at Northumberland Park Depot

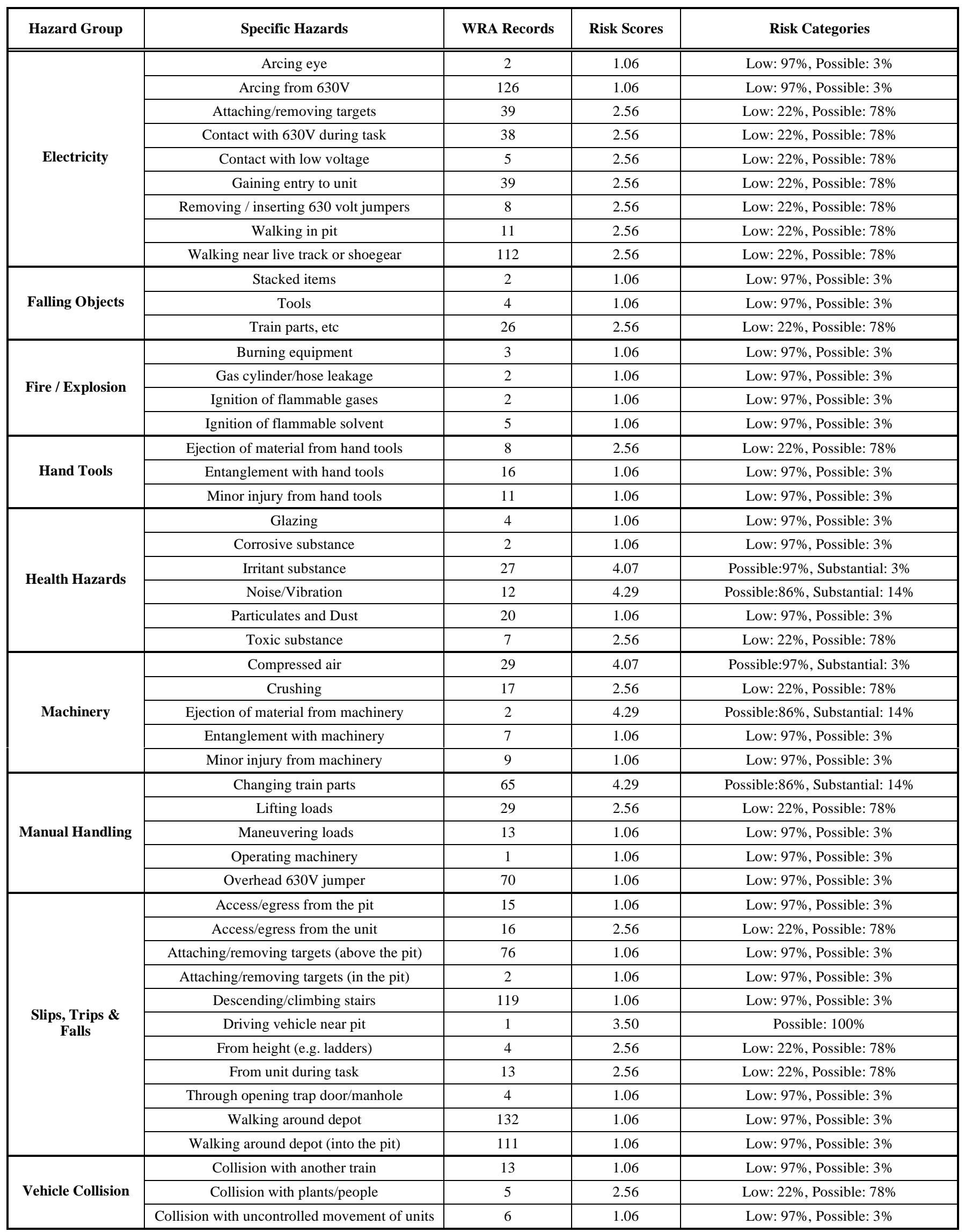


The number of WRA risk records associated with the Northumberland Park Depot is 1290. By using the proposed intelligent system, the risk score of staff risk at the Northumberland Park Depot is 4.07 and risk categories of Possible and Substantial with a belief of $97 \%$ and $3 \%$ respectively. As can be seen from Table 5 that Health Hazards, Machinery and Manual Handling hazard groups have the highest risk scores of 4.07, which belongs to Possible (97\%) and Substantial (3\%). The Fire/Explosion hazard group has the lowest risk score of 1.06 and risk categories of Low and Possible with a belief of $97 \%$ and $3 \%$ respectively.

The risks of specific hazards associated with Northumberland Park Depot are listed in Table 6. The hazards, for example, Noise/Vibration, Ejection of material from machinery and Changing train parts, have the highest risk scores of 4.29 belonging to Possible and Substantial with a belief of $86 \%$ and $14 \%$ respectively.

\section{CONCLUSIONS}

This paper presents a proposed intelligent system for railway safety risk assessment. The development of fuzzy qualitative descriptors and their MFs of FP, CS and RL expressions to quantify RLs are discussed. The relationship between the risk factors and RL expressions represented by the fuzzy rules are described.

The fuzzy reasoning approach offers a great potential in the safety risk modelling of railway systems, particularly, when the safety risk data are incomplete or there is a high level of uncertainty involved in the safety risk data. Safety risk analysis by using fuzzy reasoning approaches can formulate domain human experts' knowledge and engineering judgements. Furthermore, the knowledge base can be built by transforming information from various sources in the fuzzy logic inference process. The proposed intelligent railway safety risk assessment system can provide comprehensive results of safety risk analysis in two formats, i.e. risk score in a defined region and risk categories with a belief of percentage. The risk categories employ the qualitative descriptors that are more expressive and natural way to describe the risk issues. This can promote the understanding of risks associated with a railway system. An illustrative example of staff risk assessment at Northumberland Park Depot has been carried out to evaluate the performance of the proposed intelligent system. The results of the case study demonstrated that there are benefits to be gained by using the proposed intelligent system. The results produced from safety risk assessment provide railway engineers, maintainers, and managers with valuable information for risk response decisionmaking. The proposed intelligent railway safety risk assessment system will provide railway safety risk analysts, operators, infrastructure engineers, and managers with a method and tool to improve their safety management, and set safety standards.

\section{CONFLICT OF INTEREST}

The authors confirm that this article content has no conflict of interest.

\section{ACKNOWLEDGEMENTS}

The work described herein is part of a research project on 'An intelligent safety prediction system for rail design and maintenance' funded by the Physical Sciences Research
Council (EPSRC) under Grant no. GR/S07292 with technical support from London Underground Ltd, Tube Lines Ltd, Metronet SSL, Rail Safety \& Standard Board (RSSB), Network Rail Ltd and Serco Assurance Ltd. Their support is gratefully acknowledged.

\section{DISCLOSURE}

Part of information included in this article has been previously published in "doi: 10.1243/09544097JRRT34, Proceedings of the Institution of Mechanical Engineers, Part F: Journal of Rail and Rapid Transit 220(2), pp. 153-167, 2006"

\section{REFERENCES}

[1] M. An, Y. Chen, and C. J. Baker, "A fuzzy reasoning and fuzzyanalytical hierarchy process based approach to the process of railway risk information: A railway risk management system", Inf. Sci., vol. 181 no. 18 , pp. 3946-3966, 2011.

[2] M. An, S. Huang, and C.J. Baker, "Railway risk assessment - the FRA and FAHP approaches: a case study of shunting at Waterloo depot", In: Proc. Int. Mech. Engineers, Part F J. Rail Rapid Transit, Sage Publications: London, vol. 221, no. 3, pp. 365-383, 2007.

[3] M. An, W. Lin, and A. Stirling, "Fuzzy-reasoning-based approach to qualitative railway risk assessment", Proc. IMechE, Part F: J. Rail Rapid Transit, vol. 220 no. 2, pp. 153-167, 2006.

[4] V. A. Profillidis, Railway Management and Engineering. Ashgate: England, 2006.

[5] Railway Safety and Standard Board, Profile of Safety Risk on the UK Mainline Railway. SP-RSK-3.1.3.11, May 2003.

[6] W. Lin, M. An and A. Stirling, "Development of railway safety and risk analysis system using fuzzy reasoning approaches", In: $8^{\text {th }}$ International Railway Engineering Conference (CD format), Engineering Technics Press: Edinburgh, 2005.

[7] London Underground Limited, London Underground Limited Quantified Risk Assessment. Update 2001. 1, 2001.

[8] J. S. Jang, C. T. Sun, and E. Mizutani, Neuro-fuzzy and Soft Computing: a Computational Approach to Learning and Machine Intelligence. Prentice Hall, Upper Saddle River: New Jersey, USA, 1997.

[9] Metronet SSL, "Framework for the Assessment of HS\&E Risks". Second Metronet SSL Interim Report, 2005.

[10] R. I. Muttram, "Railway Safety's safety risk model", Proc. Int. Mech. Engineers, Part F: J. Rail Rapid Transit, Sage Publications: London: vol. 216, pp. 71-79, 2002.

[11] M. An, W. Lin, and A. Stirling, "Railway safety risk assessment using fuzzy reasoning approach", World J. Eng., vol. 2, no. 2, pp. 198-207, 2005.

[12] C. J. Bouch, C. Roberts, and J. Amoore, "Development of a common set of European high-level track maintenance cost categorise", In: Proc. Int. Mech. Engineers, Part F: J. Rail Rapid Transit, Sage Publications: London, vol. 224, no. 3, pp. 327-335, 2010.

[13] J. B. Bowles, and C. E. Peláez, "Application of fuzzy logic to reliability engineering", In: Proc. IEEE, Sage Publications: London, vol. 83 no. 3, pp. 435-449, 1995.

[14] European Standard, EN50129, "Railway applications - safety related systems for signalling", Comite European de Normalisation Electrotechnique, Brussels, May 1998.

[15] A. Kennedy, "Risk management and assessment for rolling stock safety cases", In: Proc. Int. Mech. Engineers, Part F: J. Rail Rapid Transit, Sage Publications: London, vol. 211 no. 2, pp. 67-72, 1997.

[16] W. Lin, M. An, and A. Stirling, "Railway safety assessment using fuzzy reasoning approach", In: $7^{\text {th }}$ International Railway Engineering Conference (CD Format), Engineering Technics Press: Edinburgh, 2004.

[17] L. A. Zadeh, "Fuzzy sets", Inf. Control, vol. 8, pp. 338-353. 1965.

[18] J. H. Mathews, Numeric Methods for Mathematics, Science and Engineering. $2^{\text {nd }}$ ed., Prentice Hall: Englewood Cliffs, New Jersey, 1992.

[19] O. P. Yadav, N. Singh, R. B. Chinnam, and P. S. Goel, "A fuzzy logic based approach to reliability improvement estimation during 
product development", Reliab. Eng. Syst. Safety, vol. 80, pp. 63-74. 2003.

[20] A. Sergaki, and K. Kalaitzakis, "A fuzzy knowledge based method for maintenance planning in a power system”, Reliab. Eng. Syst. Safety, vol. 77, pp. 19-30, 2002.

[21] Railway Safety and Standard Board, Guidance on the Preparation of Risk Assessment within Railway Safety Cases. Railway Group Guidance Note - GE/GN8561, June 2002.

[22] L. Wang, 1997. A Course in Fuzzy Systems and Control, Prentice Hall: Englewood Cliffs, New Jersey, 1997.

[23] K. Xu, L. Tang, M. Xie, S. L. Ho, and M. L. Zhu, "Fuzzy assessment of FMEA for engine systems", Reliab. Eng. Syst. Safety, vol. 75, pp. 17-29. 2002.
[24] J. B. Bowles, and C. E. Peláez, "Fuzzy logic prioritisation of failure in a system failure mode, effects and criticality analysis", Reliab. Eng. Syst. Safety, vol. 50, 203-213, 1995.

[25] J. M. Mendel, "Fuzzy logic system for engineering: a tutorial", Proc. IEEE, vol. 83, no. 3, pp. 345-377, 1995.

[26] Metronet SSL, "Development of Metronet Staff Risk Model”. First Metronet SSL Interim Report, 2005.

[27] European Standards, EN50126, "Railway applications - the specification and demonstration of reliability, availability, maintainability and safety (RAMS)" CENELEC, British Standards Institute: UK, September 1999.

(C) An et al.; Licensee Bentham Open.

This is an open access article licensed under the terms of the Creative Commons Attribution Non-Commercial License (http://creativecommons.org/licenses/by$\mathrm{nc} / 3.0 /$ ) which permits unrestricted, non-commercial use, distribution and reproduction in any medium, provided the work is properly cited. 